\title{
ARBITRAGE POSSIBILITIES IN BESSEL PROCESSES AND THEIR RELATIONS TO LOCAL MARTINGALES.
}

\author{
Freddy Delbaen \\ Walter Schachermayer \\ Department of Mathematics, Vrije Universiteit Brussel \\ Institut für Statistik, Universität Wien
}

\begin{abstract}
We show that, if we allow general admissible integrands as trading strategies, the three dimensional Bessel process, Bes $^{3}$, admits arbitrage possibilities. This is in contrast with the fact that the inverse process is a local martingale and hence is arbitrage free.

This leads to some economic interpretation for the analysis of the property of arbitrage in foreign exchange rates. This notion (relative to general admissible integrands) does depend on the fact, which of the two currencies under consideration is chosen as numéraire.

The results rely on a general construction of strictly positive local martingales. The construction is related to the Föllmer measure of a positive super-martingale.
\end{abstract}

\section{Introduction.}

In our paper Delbaen-Schachermayer [DS1], we showed that the inverse of the Bes $^{3}$ process, an example of a strict local martingale, doesn't allow arbitrage possibilities. In the present paper we investigate the Bes $^{3}$ process itself. The methods used in Delbaen-Schachermayer [DS1] show that with respect to simple integrands, the $\mathrm{Bes}^{3}$ process satisfies the no-arbitrage property. It is therefore not unreasonable to investigate if also with respect to general admissible integrands, the Bes ${ }^{3}$ process keeps this no-arbitrage property. We show that this is not the case, i.e. the $\mathrm{Bes}^{3}$ process permits arbitrage with respect to general admissible integrands, and we will show that there is a general statement behind this phenomenon.

1991 Mathematics Subject Classification. 90A09,60G44, 46N10,47N10,60H05,60G40.

Key words and phrases. arbitrage, Bessel processes, martingale, local martingale, equivalent martingale measure, representing measure, Föllmer measure, risk neutral measure, hedging, stochastic integration, mathematical finance.

Part of this research was supported by the European Community Stimulation Plan for Economic Science contract Number SPES-CT91-0089

Typeset by $\mathcal{A} \mathcal{M S}-\mathrm{TE}_{\mathrm{E}} \mathrm{X}$ 
We will make use of the notation and definitions as in the book by Revuz and Yor [RY]. Especially for the definition of Bessel processes, the theory of continuous martingales and Girsanov transformations, we will use this book as the basic reference.

The authors want to thank Michel Émery and Marc Yor for helpful discussions.

\section{Construction of Strictly Positive Strict Local Martingales}

Let us first introduce some notation. We will use a filtered probability space $\left(\Omega,\left(\mathcal{F}_{t}\right)_{0 \leq t \leq 1}, P\right)$, where the filtration $\mathcal{F}$ is supposed to satisfy the usual assumptions. This means that $\mathcal{F}_{0}$ contains all null sets of $\mathcal{F}_{1}$ and that the filtration is right continuous.

The continuous martingale $M$ (with respect to $P$ ), defined on the interval $[0,1]$ has value 1 at time 0 and is supposed to be $P$ uniformly integrable. Most of the time we suppose that it has a strictly positive probability to become zero. The stopping time $T$ is defined as

$$
T=\inf \left\{t \mid M_{t}=0\right\} .
$$

If the martingale does not hit zero then $T$ is simply equal to 1 . The measure $R$ is defined on $\mathcal{F}_{1}$ as the measure with Radon-Nikodym derivative equal to $M_{T}=$ $d R / d P$. From the optional stopping time theorem it follows that on $\mathcal{F}_{t}$ the RadonNikodym density is simply $M_{t \wedge T}$. We remark that if $P\left[M_{T}>0\right]<1$, the measure $R$ is only absolutely continuous with respect to $P$. A local martingale that is not a uniformly integrable martingale will be called a strict local martingale. This terminology was introduced by Elworthy, Li and Yor [ELY], where an analysis of strict local martingales is given.

We shall always denote by $N$ the process defined as

$$
N_{t}=M_{t}-\int_{0}^{t}\left(1 / M_{u}\right) d\langle M, M\rangle_{u}
$$

It follows from Lenglart's extension of the Girsanov formula (see, e.g. Revuz-Yor [RY] p.303 or [L]) that $N$ is an $R$ - local martingale and that

$$
d M_{t}=d N_{t}+\frac{1}{M_{t}} d\left\langle N_{t}, N_{t}\right\rangle
$$

is the Dob-Meyer decomposition of the semi-martingale $M$ under $R$.

The usual setting in the applications of probability theory to mathematical finance is that of a stochastic process $S$ describing the (discounted) price of a stock. A basic problem then is to decide whether there is an equivalent local martingale measure for the process $S$ and to investigate the set of all such measures (see, e.g. Delbaen-Schachermayer [DS2] and references given there).

The idea of this paper is to turn things upside down. The role of the price process $S$ will be taken by the process $M$ under the measure $R$.

If $P$ and $R$ happen to be equivalent then, of course, the process $M$ (considered with respect to $R$ ) admits at least one equivalent martingale measure, namely $P$. The interesting aspect arises if $R$ is only absolutely continuous with respect to $P$, 
but not equivalent to $P$. In this case $P$ fails to be an equivalent martingale measure for the process $M$ (considered with respect to $R$ ), as $P$ is not absolutely continuous with respect to $R$. If we can deduce from martingale representation arguments that $P$ is the only candidate for a martingale measure, then we may conclude that there is no $R$-equivalent martingale measure. As a consequence we deduce that $M$ under $R$ does not satisfy the property of No Free Lunch with Vanishing Risk, a concept introduced in Delbaen-Schachermayer [DS2] and for locally bounded semimartingales equivalent to the existence of an equivalent local martingale measure. We will show that in the case under consideration, $M$ (seen under $R$ ) allows arbitrage for general admissible integrands. As shown in Delbaen-Schachermayer [DS2] this is strictly stronger than the non-existence of an $R$-equivalent local martingale measure.

The reason for proceeding in this way lies in the fact that it is well suited to analyse Bessel processes and allows to exhibit a general phenomenon occurring in this setting. Let $B=\left(B_{t}\right)_{0 \leq t}$ be a one-dimensional standard Brownian Motion starting at $B_{0}=1$ and defined on $\left(\Omega,\left(\mathcal{G}_{t}\right)_{0 \leq t}, P\right)$. We define $T$ as the first instant the Brownian Motion $B$ hits 0 with the convention that $T=1$ if $B$ did not hit 0 before time $t=1$. The process $M$ is defined as $M=B^{T}$, the Brownian Motion stopped at time $T$. By $\left(\mathcal{F}_{t}\right)$ we denote the natural filtration generated by $M$. Clearly $M$ is the $P$-martingale associated to a $P$-absolutely continuous measure $R$ on $\mathcal{F}_{1}$ whose density is given by $M_{1}=M_{T}$. As the probability that $M_{1}$ equals zero is strictly positive, $R$ fails to be equivalent to $P$. Under the measure $R$ the continuous martingale $N$, as defined above, is a stopped Brownian Motion since $\langle N, N\rangle_{t}=\langle M, M\rangle_{t}=t$. The natural filtration, under the measure $R$, of the process $N$ is the filtration $\mathcal{F}_{t}$ augmented with the subsets of $\left\{M_{T}=0\right\}$. Under $R$, the process $M$ satisfies the differential equation

$$
d M_{t}=d N_{t}+\frac{1}{M_{t}} d t
$$

It is therefore a $\mathrm{Bes}^{3}$ process starting at the point 1.

In addition, in the present example we have martingale representation theorems at hand, which will allow us to carry out the program sketched above.

The previous description of the $\mathrm{Bes}^{3}$ process is well known, see for instance Revuz-Yor [RY] p. 294 and Ex 1.2.2, question 2 p. 419. This description is an example of a more general procedure known as the construction of the $h$-process. In Biane-Yor [BY] this construction was used to study properties of the "méandre brownien". In order to keep a more general framework and, in particular, to be able to derive results for Bessel processes of dimension $\delta \neq 3$ we place us in the more general framework of an arbitrary $P$ - absolutely continuous probability $R$ on $\mathcal{F}_{1}$ and the associated processes $M$ and $N$ defined above.

Theorem 1. If $R$ is absolutely continuous with respect to $P$ but not equivalent to $P$, then the process $1 / M_{t}$ is a $R$-strict local martingale.

Proof. Under the measure $R$, the process $M$ is a special semi-martingale that is decomposed as $M_{t}=N_{t}+\int_{0}^{t} 1 / M_{u} d\langle M, M\rangle_{u}$ into its local martingale part and its 
predictable component. Under $R$, the process $M$ is almost never zero and therefore $1 / M$ is also a semi-martingale. Stochastic calculus shows that under $R$, we have

$$
d\left(1 / M_{t}\right)=-1 / M_{t}^{2} d N_{t}
$$

and hence it is a local martingale. Since it is positive it is a supermartingale under $R$. To see that it is not a martingale, it is sufficient to remark that $E_{R}\left[1 / M_{1}\right]=$ $P\left[M_{t \wedge T}>0\right]<1$.

Remark. The passage from $P$ to $R$ poses the subsequent technical difficulty: the filtration $\left(\mathcal{F}_{t}\right)$ does not satisfy the usual assumptions with respect to $R$. But this problem is not hard to fix: the filtration $\left(\mathcal{G}_{t}\right)$ defined as $\mathcal{G}_{t}=\sigma\left(\mathcal{F}_{t}\right.$, all subsets of $\left\{M_{T}=\right.$ $0\})$ satisfies the usual assumptions. An easy exercise on monotone classes shows that for every $\left(\mathcal{G}_{t}\right)$-predictable process $H$, there is a $\left(\mathcal{F}_{t}\right)$-predictable process $K$ such that $\left\{\exists t \mid H_{t} \neq K_{t}\right\} \subset\left\{M_{T}=0\right\}$. For later use it is also useful to remark that the natural filtrations of $M$ and $N$ under $R$ are the same.

Theorem 2. If the martingale $M$ has the $\mathcal{F}$-predictable representation property with respect to $P$ then the $R$-local martingale $N$ also has the $\mathcal{G}$-predictable representation property with respect to $R$. Consequently $1 / M$ also has the $\mathcal{G}$-predictable representation property with respect to $R$.

Proof. See Th 12.22 in Jacod's book, [J].

Corollary. If $M$ is a local martingale with the $\mathcal{F}$-predictable representation property under $P$ and if $R$ and $P$ are equivalent, i.e. $P\left[M_{T}=0\right]=0$, then $1 / M$ has the $\mathcal{F}$-predictable representation property under $R$.

Before we formulate the next theorem we recall the notion of simple integrands, of general admissible integrands and of no-arbitrage. (see Delbaen-Schachermayer [DS2])

Definition. We say that a predictable process $H$ is simple if it is of the form

$$
H=\sum_{k=0}^{n-1} f_{k} 1_{\rrbracket T_{k}, T_{k+1} \rrbracket}
$$

where $0 \leq T_{0} \leq T_{1} \ldots \leq T_{n} \leq 1$ are stopping times and $f_{k}$ are $\mathcal{F}_{T_{k}}$ measurable functions. A predictable process $H$ that is $S$-integrable for a semi-martingale $S$ is called $a$-admissible (for $a \in \mathbb{R}$ ) if $H \cdot S \geq-a$. We say that $S$ satisfies the no-arbitrage property with respect to simple integrands if, for $H$ simple predictable and such that $(H \cdot S)_{1} \geq 0$ almost surely, we have that $(H \cdot S)_{1}=0$. The semimartingale $S$ satisfies the no-arbitrage property for general admissible integrands if $H$ admissible and $(H \cdot S)_{1} \geq 0$ a.s. imply $(H \cdot S)_{1}=0$. If the underlying probability measure $P$ plays a role, we add the phrase "with respect to $P$ ".

Remark. Simple integrands are not necessarily admissible.

We remark that if $S$ allows arbitrage for simple integrands, then the simple predictable process used to construct the arbitrage can be taken (see DelbaenSchachermayer [DS1]) of the form $H=f 1_{1 T_{0}, T_{1}}$ where $T_{0} \leq T_{1}$ are two stopping 
times and where $f$ is $\mathcal{F}_{T_{0}}$ measurable. When we split $f$ into its positive and its negative part, we immediately see that we can either take $f=-1$ or $f=1$.

From this it easily follows that a strictly positive process $S$ satisfies the noarbitrage property for simple integrands if and only if the process $\frac{1}{S}$ satisfies the no-arbitrage property with respect to simple integrands. We warn the reader that if we look at arbitrage with respect to admissible simple integrands the statement is no longer true. An example will be given below.

Theorem 3. If $M$ has the $\mathcal{F}$-predictable representation property for $P$ and if $P\left[M_{T}=0\right]>0$, then the process $M$ seen under $R$, admits arbitrage for general admissible integrands.

Proof. Take the real number $\alpha$ so that $f=1_{\left\{M_{T}>0\right\}}-\alpha 1_{\left\{M_{T}<0\right\}}$ satisfies $E_{P}[f]=$ 0 . Since $P\left[M_{T}=0\right]>0$, such an $\alpha$ exists and we have that $\alpha \geq 0$. By the representation property there is $H$ predictable such that $f=(H \cdot M)_{1}$ and $H \cdot M \geq$ $-\alpha$ almost surely with respect to $P$. It follows that under the measure $R$ the integrand $H$ is admissible (the process $(H \cdot M)$ is bounded from below by $-\alpha$ ) and produces an $R$ almost surely positive outcome $1_{\left\{M_{T}>0\right\}}$.

Corollary. The Bes ${ }^{3}$ process in its natural filtration permits arbitrage.

Proof. This follows easily from the theorem and the construction of the $\mathrm{Bes}^{3}$ process given above.

Remark. As shown in Delbaen-Schachermayer [DS1] the inverse of the Bes ${ }^{3}$ process satisfies the no-arbitrage property with respect to simple, not necessarily admissible, integrands. The $\mathrm{Bes}^{3}$ process therefore also satisfies the property of no-arbitrage with respect to simple integrands.

Remark. The problem whether or not $M$ is arbitrage free under $R$ is tricky and depends on the kind of arbitrage used. Under the measure $P, M$ is a uniformly integrable martingale and hence arbitrage free in any reasonable sense and in particular for simple bounded integrands. Under the measure $R$, the situation changes. The process $\frac{1}{M}$ is a $R$-local martingale and hence satisfies the no-arbitrage property with respect to general admissible integrands. Under the measure $R$, the process $M$ however might allow arbitrage opportunities with respect to simple admissible integrands. This in turn implies that $\frac{1}{M}$, a local martingale under $R$, allows arbitrage with respect to simple, not necessarily admissible, integrands. (see Delbaen-Schachermayer [DS1] for another example in this direction).

The following example is another illustration. On a probability space with a Brownian Motion $B$, endowed with the natural filtration, we define

$$
S_{t}=\exp \left(\int_{0}^{t} 1 / \sqrt{(1-u)} d B_{u}-1 / 2 \int_{0}^{t} 1 /(1-u) d u\right)
$$

We stop $S$ when it hits either 2 or 0 . Since $S_{t}$ tends to 0 almost surely when $t$ tends to 1 , the stopping time is well defined and the resulting stopped process is a bounded non-negative martingale. The measure $R$ is defined as $d R=S_{T} d P$. Under $R$, the process $S$ admits arbitrage with respect to simple admissible integrands. Indeed $S_{1}-S_{0}=1$ under $R$. The process $\frac{1}{S}$ is a strict $R$-local martingale. Under $R$ the 
outcome $\frac{1}{S_{0}}-\frac{1}{S_{1}}$ is realised by a simple integrand but it is not an outcome of an admissible integrand.

In the general setup of theorem $3, M$ satisfies the property that for stopping times $U \leq V, f=M_{V}-M_{U} \leq 0 R$ a.s. implies $f=0, R$ a.s.. We can see this as follows. The relation $f \leq 0$ implies that $\frac{1}{M_{V}}-\frac{1}{M_{U}} \geq 0 R$ a.s. But under $R$, the process $1 / M$ is a positive local martingale and hence a supermartingale. We therefore obtain $E_{R}\left[\frac{1}{M_{V}}-\frac{1}{M_{U}}\right] \leq 0$. This implies that $\frac{1}{M_{V}}-\frac{1}{M_{U}}=0 R$ a.s. and hence $f=0 R$ a.s..

To analyse the converse situation we suppose that there are two stopping times $U \leq V$ such that $R$ a.s. we have $M_{V}-M_{U} \geq 0$. By definition of $R$ and by the no-arbitrage property of $M$ (under $P$ ) we necessarily have that $\left\{M_{V}-M_{U} \leq 0\right\} \subset$ $\left\{M_{T}=0\right\}$. This means that when at time $U$ there is still a possibility to lose money, it is only due to the fact that $M$ can become zero.

Theorem 3 as well as the example given above, illustrate what can happen if we only look at survivors. In statistics one encounters the phenomenon of survivor bias when dealing e.g. with outperformance of stocks and when investigating the efficient market hypothesis. If a sample of today's stocks or mutual funds is taken and if the history of the corresponding returns is analysed, the statistician in fact only looks at survivors. The stocks, mutual funds, investment opportunities that performed very badly did not survive and the sample suffers from survivor bias. (see e.g. Ross $[R]$ ). The example given is such an illustration. By looking at the trajectories that survived we were even able to obtain arbitrage with respect to simple integrands.

Theorem 3 shows the general case. Arbitrage with respect to simple integrands is not always possible (see the case of the $\mathrm{Bes}^{3}$ process) but with respect to general integrands, arbitrage is present.

\section{Converse Theorems}

The preceding situation is more general than it first looks. This section is devoted to a converse of theorems 1 and 3 . We will show that under certain conditions, a strictly positive strict local martingale has the same distribution as the ones obtained from theorem 1 . We will also show that if $L$ is a strictly positive strict local martingale that satisfies the predictable representation property, then the conclusion of theorem 3 always holds. When we deal with the distribution of a process we mean the image measure on a natural space of trajectories. Because we also need an extension theorem for measures we need a space that is big enough.

The construction is an interpretation of the construction of the Föllmer measure of a supermartingale.(see Föllmer [F], Azéma-Jeulin [AJ] and Meyer [M]). So the methods we use are standard. However in our approach the supermartingale is a strictly positive continuous local martingale and this simplifies the construction and allows us to use a natural space of trajectories. Referring to Meyer [M] we add an extra (absorbing) point to the state space $\mathbb{R}_{+}$, i.e. we will work with the compact space $[0, \infty]$.

The space of trajectories is the space $C_{\infty}[0,1]$ of continuous paths $\omega$ defined on the time interval $[0,1]$ with values in $[0, \infty]$ and with the extra property that if $\omega(t)=\infty$ then $\omega(s)=\infty$ for all $s \geq t$. The set $C_{\infty}$ is a Borel set of the space of all continuous functions from $[0,1]$ into $[0, \infty]$ endowed with the topology of uniform 
convergence.

The evaluation functionals are denoted $L_{t}$, hence $L_{t}(\omega)=\omega(t)$. They take values in $[0, \infty]$. The filtration generated by the process $\left(L_{t}\right)_{0 \leq t \leq 1}$ is denoted by $\mathcal{H}_{t}^{\circ}$, the superscript meaning that we do not saturate this filtration in order to satisfy the usual conditions. The results of Föllmer, $[\mathrm{F}]$ and especially the presentation in Meyer, $[\mathrm{M}]$ can be translated into the following theorem. We only give a sketch of the proof.

Theorem 4. If $R$ is a measure on $C[0,1]$ such that $L$ is a strictly positive strict local martingale, then

(i) there is a probability measure on $C_{\infty}[0,1]$ such that $M=\frac{1}{L}$ is a $P$ martingale.

(ii) we may choose $P$ in such a way that the measure $R$ is absolutely continuous with respect to $P$ and its Radon-Nikodym derivative is given by $d R=M_{1} d P$.

(iii) if $L$ has the predictable representation property with respect to $R$ then $M$ has the predictable representation property with respect to $P$. In this case the process $\frac{1}{L}$, seen under the measure $R$, allows arbitrage with respect to general admissible integrands.

Proof. The measure $R$ is defined on $\mathcal{H}_{1}^{\circ}$ and is such that the process $L$ is a strictly positive strict local martingale. The strict positivity of $L$ results in $R\left[L_{1}=0\right]=0$. The stopping times $T_{n}$ are defined as the first hit of the level $n, T_{n}=\inf \left\{t \mid L_{t} \geq\right.$ $n\} \wedge 1$. It is easy to see that $T_{n}$ is a stopping time for the filtration $\mathcal{H}^{\circ}$. Also $\lim _{n \rightarrow \infty} T_{n}=T$, where $T=\inf \left\{t \mid L_{t}=\infty\right\} \wedge 1$. The sigma algebras $\mathcal{H}_{T_{n}}^{\circ}$ are increasing and their union generates $\mathcal{H}_{T}^{\circ}=\mathcal{H}_{1}^{\circ}$.

The stopped processes $L^{T_{n}}$ are bounded continuous martingales for the measure $R$. On each of the sigma algebras $\mathcal{H}_{T_{n}}^{\circ}$ we define the measure $P_{n}$ as $d P_{n}=L_{T_{n}} d R$. By the martingale property we have that $P_{n+1}$ restricted to $\mathcal{H}_{T_{n}}^{\circ}$ is precisely $P_{n}$. We obtain in this way an additive set function $P$ defined on $\bigcup_{n \geq 1} \mathcal{H}_{T_{n}}^{\circ}$. This additive set function is sigma additive and can be extended to a true probability measure on $\mathcal{H}_{1}^{\circ}$.

Theorem 5. Let $\left(\Omega,\left(\mathcal{F}_{t}\right)_{0<t<1}, R^{\prime}\right)$ be a probability space with a filtration that satisfies the usual assumptions. Let $L^{\prime}$ be a strictly positive, continuous, strict local martingale. Assume that $\mathcal{F}$ is the natural filtration of $L^{\prime}$ and that $L^{\prime}$ has the predictable representation property. Under these assumptions, the process $1 / L^{\prime}$ allows arbitrage with respect to general admissible integrands.

Proof. We define a mapping $\Phi: \Omega \rightarrow C_{\infty}$ as follows $\Phi(\omega)(t)=L_{t}^{\prime}(\omega)$. Since $L^{\prime}$ is almost everywhere continuous the mapping $\Phi$ is well defined (if needed we first throw away a set of measure zero). The mapping $\Phi$ is measurable and induces a measure $R$ on $\mathcal{H}_{1}^{0}$. We now apply the theory above and since the filtration $\mathcal{F}$ satisfies the usual assumptions we see that for each $t$ the mapping $\Phi$ is measurable for the couple $\mathcal{F}_{t}-\mathcal{H}_{t}^{R}$. The process $L$ defined on $C_{\infty}$ is strictly positive, it is a strict local martingale and has the predictable representation property. (Here we use that $\mathcal{F}$ is the natural filtration generated by $L^{\prime}$ ). We therefore obtain an $\mathcal{H}^{R}$-predictable process $H$ that is admissible and that produces an arbitrage opportunity. The mapping $H^{\prime}=H \circ \Phi$ is $\mathcal{F}$-predictable, is admissible and produces arbitrage for the original process $1 / L^{\prime}$. The proof is now complete. 


\section{Bessel processes}

In this section we will apply the previous theorems to the particular case of Bessel processes of dimension $\delta>2$. (from now on $\delta$ will denote a real number that is strictly greater than 2). It is known that if $X$ is a Bessel process of dimension $\delta$, starting at $X_{0}=1$, then $L=X^{2-\delta}$ is a strictly positive strict local martingale (see Revuz-Yor [RY] p.418 ex 1.16). It is easy to see that the local martingale $L$ has the predictable representation property with respect to the natural filtration generated by $X$. Also the method of Delbaen-Schachermayer [DS1] for the case $\delta=3$ can be adapted and this yields that $L$ satisfies the no-arbitrage property with respect to simple integrands. The results of the preceding section therefore immediately yields the following:

Theorem 6. If $\delta>2$ and $X$ is a Bessel process of dimension $\delta$, then $L=X^{2-\delta}$ is a local martingale such that $\frac{1}{L}$ allows arbitrage with respect to general admissible integrands. The processes $L$ and $\frac{1}{L}$ do not allow arbitrage with respect to simple predictable integrands.

Although the preceding result is satisfactory for applications in finance, it would be nice if we could give an interpretation of the martingale $M$ needed to construct $L$. More precisely we want to make the construction of the preceding section more transparent. The main ingredient for this is the Girsanov transformation for Bessel processes. The theory is developed by Yor $[\mathrm{Y}]$ and in particular the formula (2.c) there, relates Bessel processes from dimension $\delta>2$ with Bessel processes of (possibly negative) dimension $4-\delta$. An application of these results immediately gives:

Theorem 7. If $\eta<2$ and under the measure $P, X$ is a Bes ${ }^{\eta}$ process starting at 1 and stopped at the first time $T$ when $X$ hits zero, then under the measure $R$ defined as $d R=X_{T}^{2-\eta} d P, X$ is a Bes ${ }^{4-\eta}$ process.

For $\delta=3$ we find the following situation. Let $M$ be a Brownian Motion started at $M_{0}=1$ and stopped at zero (i.e. a Bes ${ }^{1}$ stopped at zero). The process $M$ under the measure $d R=M_{1} d P$ is a Bes $^{3}$ process. Theorem 5 now shows that $M$ allows arbitrage for general admissible integrands. This result was the main reason for developing the theory of sections 1 and 2. Again the construction is an example of an $h$-process.

\section{Additional Comments.}

We gave one way to construct strictly positive strict local martingales $X$ such that $\frac{1}{X}$ allows arbitrage opportunities. Financially this means that betting on the exchange rate ECU/ $\$$ does not yield arbitrage opportunities for a European citizen, but for an American citizen, there are such possibilities. This is counter-intuitive but there is an explanation. For simple predictable strategies there are no arbitrage profits for both agents. When general admissible integrands are allowed then one of the agents can make profits. The reason can be found in the restriction that the strategy has to be admissible. This restriction is not independent of the change of currency. So one agent can use admissible strategies (buying and selling) that have no equivalent admissible translation (selling and buying) for the other agent.

In Delbaen-Schachermayer [DS3] we discuss the arbitrage property when the numéraire is changed. The results given there extend the previous results. The 
method however is related to our paper [DS2] and is more involved than the construction of strict local martingales given here.

We finally remark that Theorem 5 can be proved directly, i.e. without using the projective limit construction. Of course proceeding that way does not indicate how strict local martingales arise in a natural way. We also remark that the counterexample constructed in [S] or [DS4] yields a continuous local martingale $L$ and a uniformly integrable strictly positive martingale $Z$ such that $L Z$ is a uniformly integrable martingale. Since $Z=(1 / L)(L Z)$ is a martingale, we find that $1 / L$ has an equivalent martingale measure. This example shows that in theorem 5 , the hypothesis that $L$ has the predictable representation property cannot be dropped.

\section{REFERENCES}

[AJ] J. Azéma et T. Jeulin, Précisions sur la mesure de Föllmer, Ann. Inst. Henri Poincaré Section B XII (1976), 257-283.

[BY] Ph. Biane and M. Yor, Quelques Précisions sur le Méandre Brownien, Bull. Sc. Math, $2^{e}$ série 112 (1988), 101-109.

[DS1] F. Delbaen and W. Schachermayer, Arbitrage and Free Lunch with Bounded Risk for unbounded continuous processes, Mathematical Finance 4 (1994), 343-348.

[DS2] F. Delbaen and W. Schachermayer, A General Version of the Fundamental Theorem of Asset Pricing, Mathematische Annalen 300 (1994), 463-520.

[DS3] F. Delbaen and W. Schachermayer, The No-Arbitrage Property under a Change of Numéraire, to be published in Stochastics and Stochastic Reports.

[DS4] F. Delbaen and W. Schachermayer, A simple counter-example to several problems in the theory of asset pricing, which arises generically in incomplete markets., forthcoming.

[ELY] D. Elworthy, X.-M. Li and M. Yor, The Importance of Strict Local Martingales, forthcoming.

[F] H. Föllmer, The Exit Measure of a Supermartingale, Zeitschrift für Wahrscheinlichkeitstheorie und verwandte Gebiete 21 (1972), 154-166.

[J] J. Jacod, Calcul Stochastique et problèmes de martingales, Springer Verlag, Berlin, Heidelberg, New York, 1979.

[L] E. Lenglart, Transformation des martingales locales par changement absolument continu de probabilités, Zeitschrift für Wahrscheinlichkeitstheorie und verwandte Gebiete 39 (1977), 65-70.

[M] P. A. Meyer, La mesure de Föllmer en théorie de surmartingales, Sém. de Probabilités VI, Lecture Notes in Mathematics 258, Springer, Heidelberg, Berlin, New-York, 1972.

[R] S. Ross, Topics in Finance, Talk held during the "6.Tagung Geld, Finanzwirtschaft, Banken und Versicherungen", Karlsruhe, 1993.

[RY] D. Revuz and M. Yor, Continuous Martingales and Brownian Motion, Springer Verlag, Berlin Heidelberg New York., 1991.

[S] W. Schachermayer, A Counterexample to Several Problems in Mathematical Finance, Mathematical Finance.

[Y] M. Yor, On some Exponential Functionals of Brownian Motion, Advances in Applied Probability 24 (1993), 509-531.

Vrije Universiteit Brussel, Pleinlaan 2, B-1050 Brussels Belgium

E-mail address: fdelbaen@tena2.vub.ac.be

Universität Wien, Brünnerstrasse 72, A-1210 Wien Austria

E-mail address: wschach@ stat1.bwl.univie.ac.at 